\title{
Maximum entropy method for biodiesel spray droplet distribution
}

\author{
Ruslana Kolodnytska*, Sergii Skurativskyi, Pavel Moskvin \\ Zhytomyr State Technological University, Zhytomyr, 10005, Ukraine \\ *Corresponding author: ruslanakolod@gmail.com
}

\begin{abstract}
The efficiency of combustion process in diesel engine depends on the spray characteristics. The most important of them are droplet size and velocity distributions. There are four methods which are used for describing the droplet size distributions: empirical, maximum entropy formalism (MEF), discrete probability function (DPF) and stochastic method. The MEF assumes that spray formation is a random process that can be described using the principle of maximum entropy. DPF method is a combination of random and non-random processes when the drop-size distribution appears from fluctuations in the initial conditions. Under the DPF approach the spray formation is divided into following steps: liquid breakup, ligaments separation, breakup of ligaments into fragments, fragment breakup into droplets. The stochastic breakup model assumes that the probability of formation of daughter droplet breakup size is independent of its parent size (a fractal scaling of breakup has been identified). This paper presents an investigation into the application of MEF model for distribution of biodiesel droplets. We used the model approach with the constraints: normalization, mass conservation, momentum conservation and surface energy conservation. The resulting probability density function (PDF) for velocity and droplet size is obtained by maximizing the Shannon entropy. We also used the new numerical algorithm to improve the model accuracy. The PDF for droplets diameters with different Weber numbers were calculated for both diesel and biodiesel fuels. The MEF predictions were compared against the experimental data for diesel and biodiesel droplet distribution with different injection pressure. According to the maximum entropy method, the influence of fuel thermodynamic properties on the parameters of drop-size and velocity distribution function for fuel sprays has been analysed.
\end{abstract}

\section{Keywords}

Maximum entropy method, distribution function, spray, biofuel, biodiesel.

\section{Introduction}

The efficiency of combustion process in diesel engine depends on the spray characteristics. The most important of them are droplet size and velocity distributions [1]. There are four methods which are used for describing the droplet size distributions: maximum entropy formalism (MEF), discrete probability function (DPF), stochastic and empirical methods [2]. The MEF assumes that spray formation is a random process that can be described using the principle of maximum entropy [3,4]. This method is useful for processes where the secondary atomization were dominated. DPF method is a combination of random and non-random processes when "the drop size distribution arises from fluctuations in the initial conditions" [2]. Under the DPF approach the spray formation is divided into following steps: liquid breakup, ligaments separation, breakup of ligaments into fragments, fragment breakup into droplets [2]. Sovani et al. [3] showed that the fluid physical properties (density, viscosity and surface tension) have a negligible effect on the droplet distribution in contrast to the velocity fluctuations which has a bigger impact [2]. The stochastic breakup model assumes that the size of daughter droplets formed by the breakup of its parents is stochastically independent of the parent droplets size. Using this approach fractal scaling of breakup has been observed by several authors [5].

At the same time, the thermodynamic description of the fuel sprays can be achieved on the basis of more simple models for spray formations, so-called equilibrium models. The advantage of equilibrium counterparts for spray modelling is the possibility to reduce the number of models' parameters in comparison with the kinetic models. Moreover, the smaller number of governing parameters simplifies the interpretation of the results and identification of main thermodynamic parameters determining the final state of sprays.Among the equilibrium models describing the spray formation it is worth to distinguish the models derived within the framework of maximum entropy method. The natural constraints supporting the existence of local maximum for the entropy of droplet system are the conservation laws for mass, momentum, and energy. As a result, the application of maximum entropy method gives us the drop-size and velocity distribution which obeys the conservation laws and provides the maximum value of system's entropy.

The classical methods to predict the drop-size and velocity distribution are empirical ones that include log-normal, Rosin-Rammler, Nukiyama-Tanasawa distributions, etc. [4]. Nonetheless, some limitations in using these models have to be considered even though attempts have been made by adding more parameters [6]. We have been 
trying to use Rosin-Rammler approximation as well as others mentioned, to fit the experimental data $[7,8]$ for the biodiesel droplet diameter distribution but we have not got any good satisfying results. That is why we have decided to switch to using the MEF method following the assumption stated in [6]: "...former studies indicate that the MEF shows good prediction ability“.

Biofuels have been developed in order to reduce the dependence on crude oil as an alternative to the diesel fuel. The most prominent among fuels that derived from biological sources is biodiesel [9]. But the major problem of using biodiesel is their higher viscosity which leads to "larger drops when atomized and hence lower combustion performance" [10]. To create new type of biodiesel (for example, hemp biodiesel [11, 12]) we need to know not only the value of mean droplet diameters but also the spray droplet distribution.

This paper presents an investigation of the application of MEF model for distribution of diesel, biofuels (vegetable oil and diesel blends) and biodiesel droplets. We used the model from [4] with the following constraints: normalization, mass conservation, momentum conservation and surface energy conservation. Supposing that these processes are essentially characterized with the drop-size and velocity distribution of droplets, we are going to analyse the influence of thermodynamic parameters of fuel sprays on the sizes and velocities of droplets in the sprays.

\section{Material and methods}

\subsection{Model of maximum entropy}

According to the maximum entropy method, the process of spray formation is considered as the transition from one equilibrium state to another. To identify the initial state of the system, let us choose the state of liquid on the exit of injector, whereas the final equilibrium state corresponds to the state when the whole accessible volume is filled with the droplets of the spray.

Thus, within the chosen approach and using the drop-size and velocity probability distribution function $f(\bar{D}, \bar{u})$ for droplets, the equations for conservation of mass (1), momentum (2), and energy (3) can be written in the following dimensionless form [1, 4]:

$$
\begin{aligned}
& \iint f \bar{D}^{3} d \bar{u} d \bar{D}=1+\bar{G}_{m}, \\
& \iint f \bar{D}^{3} \bar{u} d \bar{u} d \bar{D}=1+\bar{G}_{m v}, \\
& \iint f\left(\bar{D}^{3} \bar{u}^{2}+B \bar{D}^{2}\right) d \bar{u} d \bar{D}=1+\bar{G}_{e}, \\
& \iint f d \bar{u} d \bar{D}=1,
\end{aligned}
$$

where $W e=\frac{\rho U_{0}^{2} \cdot D_{30}}{\sigma}$ is the Weber number, $D_{30}$ is mass mean diameter of droplets,

$$
B=\frac{12}{W e}, \bar{G}_{m}=\frac{G_{m}}{\dot{m}_{O}}, \bar{G}_{m v}=\frac{G_{m v}}{\dot{J}_{0}}, \bar{G}_{e}=\frac{G_{e}}{\dot{E}_{o}}, \bar{D}=D / D_{30}, \dot{E}_{o}=\dot{m}_{o} U_{0}^{2}, \bar{V}=\frac{V}{V_{m}}, \bar{u}=\frac{u}{U_{0}}, V_{m}=\frac{\dot{m}_{O}}{\rho \dot{n}} \text {. }
$$

Note that the function $f(\bar{D}, \bar{u})$ is the probability of finding a droplet with the volume $V$ and velocity $u$ and it satisfies the normalization condition (4). Equation (3) shows that the droplet of spherical form possesses the kinetic and surface energies. The latter is proportional to the area of droplet surface and surface tension.

According to the classic dependence between entropy $S$ of the system and the probability $f$ of microstates $[1,4]$, the following relation holds:

$$
S=-k_{B} \cdot \iint f \cdot \ln (f) d \bar{V} d \bar{u}
$$


We thus arrive to the mathematical problem of finding the maximal value of the entropy $S$ for droplet system when the auxiliary constraints (1) - (4) are fulfilled. This problem is solved by the Lagrangian multiplier method, application of which gives the following form for the probability density function $f$ :

$$
f(\bar{D}, \bar{u})=3 \bar{D}^{2} \exp \left[-\alpha_{0}-\alpha_{1} \cdot \bar{D}^{3}-\alpha_{2} \cdot \bar{D}^{3} \cdot \bar{u}-\alpha_{3}\left(\bar{D}^{3} \cdot \bar{u}^{2}+B \cdot \bar{D}^{2}\right)\right] \text {. }
$$

Therefore, our studies are reduced to the analysis of equations (1) - (6), while the input data for them are the sets of thermodynamic parameters of different fuels and injector operating conditions.

\subsection{Thermodynamic parameters of spray systems}

Let us first to do some analysis of the equations above and choose the thermodynamic parameters of fuels we are interested in. We also neglect the reducing of liquid mass due to evaporation. In this case it is possible to assume that in equation (1) the quantity $\bar{G}_{m}$ vanishes: $\bar{G}_{m}=0$. Similar situation concerning the additional momentum and energy sources in a spray is observed.

Note also that the momentum and energy sources in equations (2) and (3) are caused by the effects of interaction between phases. In this paper we take into account the processes of transferring between liquid and gas, for instance, the frictional effects. From equations (1) - (3) also it follows that the mechanism of liquid atomization is not very important, whereas the energy and momentum losses play a crucial role.

The arguments presented above allow us to develop an informative and efficient approach by reducing the number of governing parameters in the problem. The system (1) - (4) was analysed with different values of parameters $\bar{G}_{m v}$ and $\bar{G}_{e}$. These parameters can be regarded as the adjustable parameters derived during the matching the theoretical and experimental data. It is also safe to assume that $\bar{G}_{m N}=\bar{G}_{e}$ in specific calculations. Hence, system (1) - (4) possesses only one parameter, i.e. the Weber number defining the type of system's solution and, consequently, the type of distribution.

We also used the new numerical algorithm to improve the model accuracy. Efficiency of our mathematical and numerical models was checked by means of comparison with the results presented in [4].These results are concerned with the process of water spray formation when the Weber number is fixed at $W e=311$. For clarity, both our calculations and experimental data from [4] are given in Fig. 1. Next we are going to study the profiles of probability density function derived at different values of parameters $W e, \bar{G}_{m v}$ and $\bar{G}_{e}$.

\section{Results and discussion}

\subsection{PDF for spray with different We numbers}

The main results of the spray modelling are presented in Figures 1-3. In particular, the profiles for probability density function of normalized diameter of droplets are plotted in Fig. 1, when the Weber number is varied. During these calculations, the energy and momentum losses due to the friction are fixed as follows: $\bar{G}_{e}=0$ and $\bar{G}_{m N}=-0.017$, respectively.

As follows from Fig. 1, the process of fuel spray formation, when the Weber number increases from 100 to 1000 , causes the displacement of profiles for the probability density function. The direction of this deviation corresponds to the conditions of the formation of larger droplets.

We also found some specifics in the drop-size distributions at We $>1000$. It turned out in Fig. 1 that when increasing the Weber number of more than 1000 that would not have any significant effect on the profiles of the probability density function. It means that increasing the mean nozzle exit velocity $U_{O}$ influences on the geometric parameters of droplet system up to certain threshold of Weber number (in Fig. 1 We is less than 800). Therefore, we do not expect the essential variation of droplets sizes due to an increase of the spraying velocity at We $>1000$. As it follows from Fig. 1, the position of the maximum of the probability density function, i.e. the most probable size of droplet in the system, depends on the Weber number essentially. Moreover, when the Weber number increases, we observe the distinct displacement of the maximum in the domain of larger droplets.

Under the typical conditions of spray formation at low injection pressure [7], the fuels spray with the compositions varying from the diesel to biodiesel will have the Weber numbers less 1000 . Note, that in this work we calculated the Weber number based on $D_{30}$ rather than based on the nozzle diameter as in [7]. Fig. 1 also shows the increase of the most probable diameter of droplets from $0.25 \cdot \bar{D}$ to $0.5 \cdot \bar{D}$ when we use biodiesel instead diesel. 
This theoretical result is confirmed by the experimental evidences. It is known [13] that the mean size of droplets for the biodiesel spray is larger than for the diesel fuel spray.

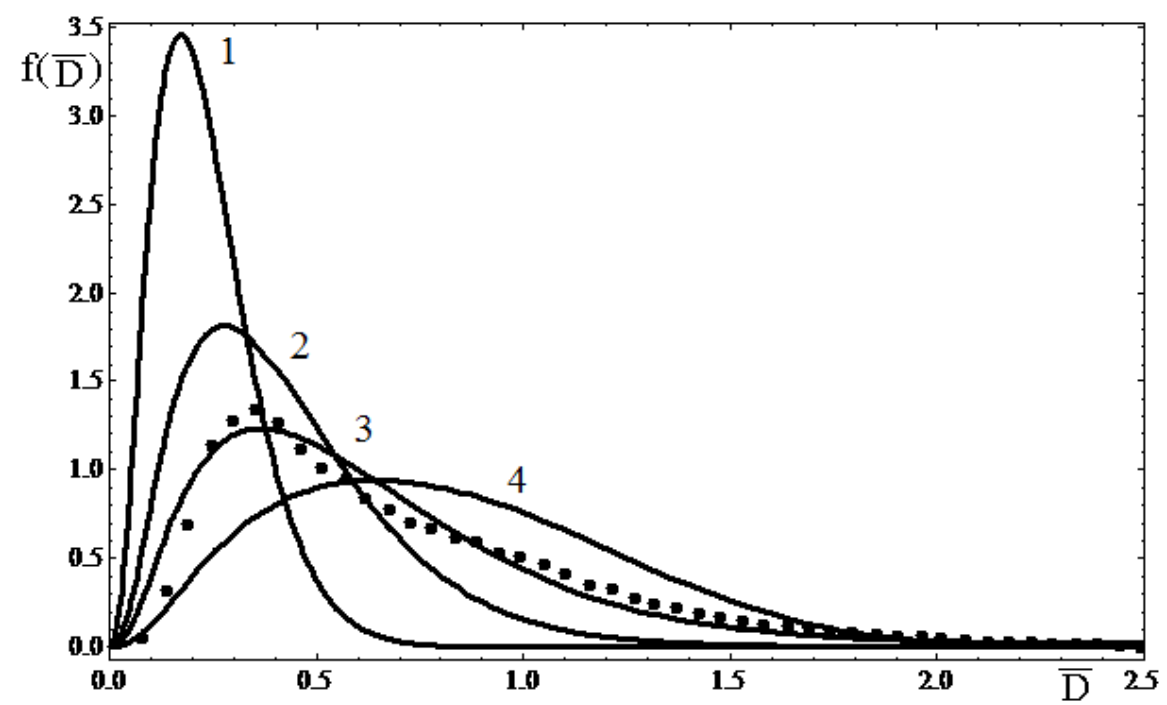

Figure 1. Probability density distribution for normalized droplet diameters at different Weber numbers: $1-$ We $=150$

$2-W e=220 ; 3-W e=311 ; 4-W e=1000$. Experimental data is taken from [13] for the water spray.

\subsection{PDF for biofuels spray (diesel and vegetable oil blends)}

The theoretic curves and experimental results [13] in the form of distributions of droplet diameters for the sprays from the blends of diesel fuel with rapeseed oil are presented in Fig. 2.

To process the experimental data [13] we need to know the fuel density and the surface tension for fuel blends. To estimate them, the mixture rules from [12] are applied to initial components of fuel blends. We also need to know the Weber numbers and the parameters $\bar{G}_{m N}, \bar{G}_{e}$ corresponding to the momentum and energy sources.

Note that the mean nozzle exit velocity $U_{0}$ gives the essential uncertainty in the final value of Weber number. It is natural that this velocity depends on the nozzle design, its operational parameters (e.g. injection pressure), and the thermodynamic properties of fuels. However in the case of diesel, the fuel density is the only fuel property that is included in the injection velocity calculation. To increase accuracy the instantaneous injection velocity was defined from transient experimental mass profile [14]. Due to the significant amount of factors defining the biofuels injection, it is difficult to make a reliable theoretical estimation of the considered parameter for the experimental equipment [13].

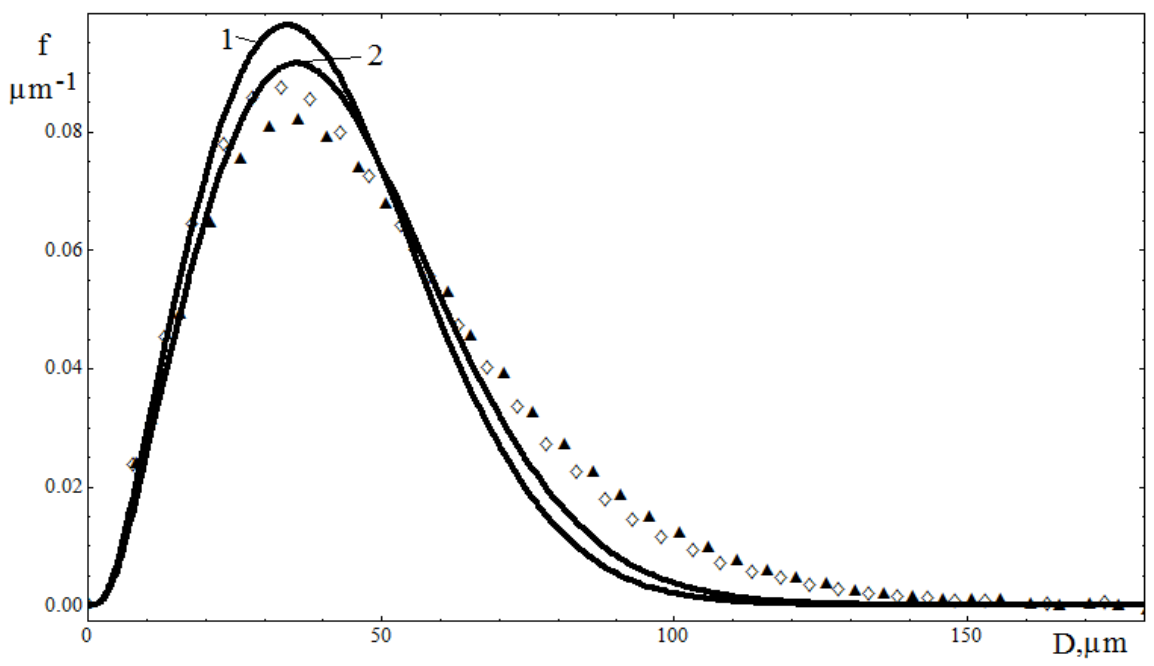

a) 


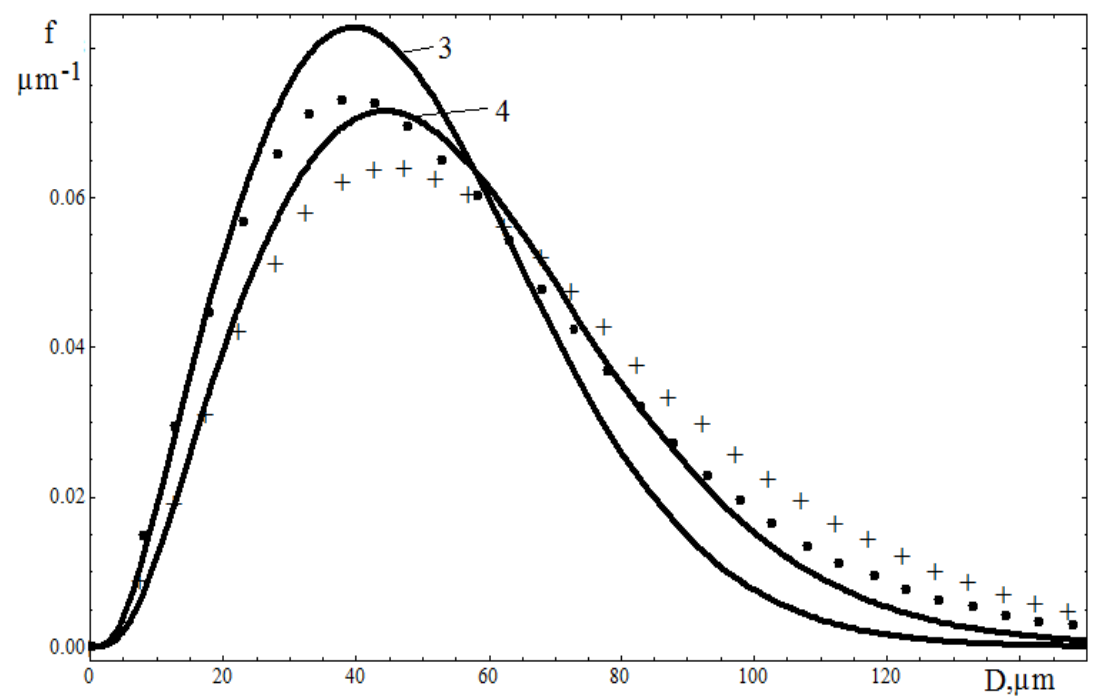

b)

Figure 2. Probability density function for the droplet diameters for diesel fuel and its blends with rapeseed oil (RO)

a) 1 - diesel fuel (DF): $W e=900, \bar{G}_{m v}=\bar{G}_{e}=-0.017$;

$2-20 \% \mathrm{RO}$ and $80 \% \mathrm{DF}: W e=825, \bar{G}_{m v}=\bar{G}_{e}=-0.018$;

$\diamond, \boldsymbol{\Lambda}$ - experimental data is taken from [13] for different compositions of fuel 1 and 2 , respectively.

b) $3-30 \% \mathrm{RO}$ and $70 \% \mathrm{DF}: \mathrm{We}=750, \overline{\mathrm{G}}_{\mathrm{mv}}=\overline{\mathrm{G}}_{\mathrm{e}}=-0.020$;

$4-40 \% \mathrm{RO}$ and $60 \% \mathrm{DF}: \mathrm{We}=450, \overline{\mathrm{G}}_{\mathrm{mv}}=\overline{\mathrm{G}}_{\mathrm{e}}=-0.032$;

,$+ \boldsymbol{\square}$ - experimental data is taken from [13] for different compositions of fuel 3 and 4 , respectively.

At the same time it is well-known that a neat vegetable oil has a higher viscosity (usually $28-40 \mathrm{~mm}^{2} / \mathrm{s}$ ) than diesel which leads to an injector coking due to poorer atomization [9]. Transesterification of the oil reduces the viscosity; biodiesel viscosity (usually $4-5 \mathrm{~mm}^{2} / \mathrm{s}$ ) is closer to diesel one [9]. According to an experimental study at Brighton University, UK, the diesel fuel exit velocity was $43.7 \mathrm{~m} / \mathrm{s}$ and the leading edge velocity of biodiesel jet was 28.3 $\mathrm{m} / \mathrm{s}$ at injection pressure $40 \mathrm{MPa}$ [7]. The same tendency was observed in another study of biodiesel - see [15], when at the start of the injection the first part of the spray (before break-up, near the orifice exit) with biodiesel seems to travel slower than the diesel fuel [15]. It was found that viscosity of the biodiesel has a strong effect on the drop size distribution and droplet mean diameters [16]. So, the viscosity and surface tension of biodiesel appear to play a dominant role on frictional losses in the nozzle [7, 15].

Thus we can assume that the mean nozzle exit velocity in case of biodiesel fuels will be less than of diesel fuel and the Weber numbers would decrease for biodiesel comparing with diesel fuels. We used this statement for low injection pressure biodiesel spray (see in Fig. 1). Also, we can assume that the Weber numbers should decrease when the fraction of vegetable oil increases (see in Fig. 2).

Increasing the fuel viscosity when the rapeseed oil is added affects the general momentum and energy consumption. It means that the parameters corresponding to the momentum and energy sources, $\bar{G}_{m v}, \bar{G}_{e}$, should also depends on types of fuels. In this case one can state that the amount of losses of momentum and energy grows when the fraction of vegetable oil in fuel blend increases. From this it follows that $\bar{G}_{m v}, \bar{G}_{e}$ are negative quantities the absolute values of which increase when the viscosity of fuel mixture grows. Thus, the assertions presented above allow us to justify the following approach for the theoretical description of experimental dependences from [13].

We are going to estimate the Weber numbers $(\mathrm{We})$, momentum and energy source terms $\left(\bar{G}_{m v}, \bar{G}_{e}\right)$ satisfying the proper conditions for the description of experimental data [13]. Using these quantities and solving the system (1) - (4), the identification of probability density function (6) is carried out. The profiles of these functions are plotted in Fig. 2 for different fuel blends.

Analysing the results, we elucidated that among $\bar{G}_{m N}, \bar{G}_{e}$, the parameter $\bar{G}_{m N}$ impacts the most significantly on the profiles of probability density functions. So, we put $\bar{G}_{m v}=\bar{G}_{e}$ in the final analysis. The following numerical analysis showed that this assumption does not lead to the loss of generality, but reduces the number of adjustable parameters and simplifies the interpretation of obtained results. 
It is worth also to note that there is a satisfactory matching between theoretical and experimental data in Fig. 2 . It is important to emphasize that the values of Weber numbers and source terms for momentum and energy derived during the numerical procedure and presented in the legends in Fig. 2 are physically acceptable and satisfy the requirements stated above.

\subsection{PDF for biodiesel spray}

Fig. 3 shows the drop-size probability density for diesel and biodiesel fuels when experimental data [8] were fitted by the MEF method using equations (1)-(6). This case is close to realistic diesel engine conditions when the injection pressure is $100 \mathrm{MPa}$. In this case diesel fuel has emerged from the nozzle orifice with a velocity of 100 $\mathrm{m} / \mathrm{s}$ as ultra-high-speed videos showed [7]. These conditions indicate that the flow is both turbulent and in atomisation regime [7]. We have analysed two cases for the droplet size distribution for both diesel and biodiesel: 1) at distance of $10 \mathrm{~mm}$ from nozzle exit (see [8] for experimental data), and 2) at distance of $15 \mathrm{~mm}$ from nozzle exit (Fig. 3). We assumed that biodiesel has lower mean injection velocity $U_{0}$ than diesel but this difference is compensated by the higher value of $D_{30}$ for biodiesel. As a result, this leads to the same values of Weber number (We $=2500$ ) for diesel and biodiesel.

At distance of $15 \mathrm{~mm}$ we found that the better agreements against the experimental data were $\bar{G}_{m v}=-0.0035$, $\bar{G}_{e}=0$ for diesel; and $\bar{G}_{m v}=-0.00375, \bar{G}_{e}=0$ for biodiesel (Fig. 3). At distance of $10 \mathrm{~mm}$ our calculations show the same result as for diesel at distance of $15 \mathrm{~mm}$. This case wasn't presented in Fig.3. The difference in momentum sources $\bar{G}_{m v}$ between diesel and biodiesel may be related to different viscosity of fuels.

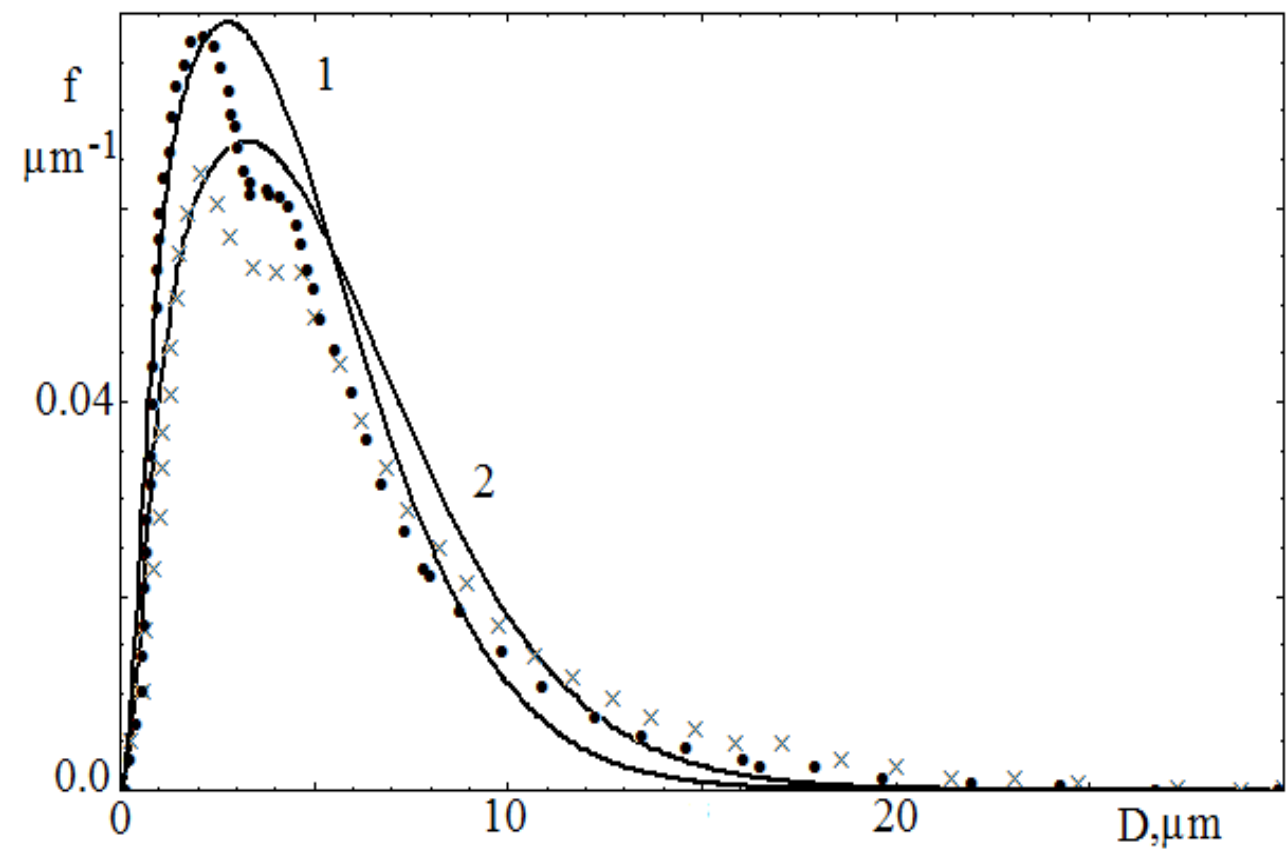

Figure 3. Probability density functions of the droplet diameters at distance of $15 \mathrm{~mm}$ from nozzle exit at We=2500:

1 - for diesel fuel $\bar{G}_{m v}=-0.0035, \bar{G}_{e}=0$

2 - for biodiesel fuel $\bar{G}_{m v}=-0.00375, \bar{G}_{e}=0$

Experimental data for diesel $(\bullet)$ and biodiesel $(x)$ are taken from [8]

At $10 \mathrm{~mm}$ from the nozzle exit, the intersection of experimental droplets size distribution of biodiesel and diesel occurred at the $8 \mu \mathrm{m}$ bin (see Fig. 7a, [8]). At $15 \mathrm{~mm}$ away from nozzle the experimental droplet distribution for RME was more skewed to the right comparing with the diesel with intersection at $5 \mu \mathrm{m}$ bin (see Fig. 7b, [8]).

Almost the same tendency for biodiesel was observed in experiments [10] with a solid-cone pressure-swirl atomizer at $30 \mathrm{~mm}$ from the nozzle exit when biodiesel PDF also was skewed to the right. At $70 \mathrm{~mm}$ from nozzle exit, along injector axis, the drop size distributions of biodiesel were not significantly different to that of diesel fuel [10]. But a big difference between diesel and biodiesel in the area between the core and the edge of spray at the radial position of $1 \mathrm{~cm}$ from the centre has been found in [10].

So, as expected from the physical properties of diesel and biodiesel fuels, both the theoretical and the experimental droplet size distributions show that biodiesel produces a higher number of larger droplets than diesel 
fuel [8]. This tendency is more profound in the sections far from the nozzle and in the area between the core and the edge of spray.

It is also important to notice that for realistic diesel condition when evaporation has place both the spray phenomena and mean nozzle exit velocity of fuel depend on the cavitation process. The detailed velocity research for biodiesel in this case was carried out in [17]. In case of cavitation, when the pressure at the vena contracta was lower than the vapour pressure new discharge coefficient was proposed [17]. If the flow at the nozzle exit is turbulent, the injection velocity is calculated using the volumetric mean velocity and the initial droplet size is equal to the nozzle hole diameter. In case of the super cavitation in the nozzle flow, the cross-sectional area of the initial blob was more diminished in size than the diameter of nozzle exit [17].

\section{Conclusions}

The maximum entropy method was applied for the droplet distribution of diesel, biodiesel and biofuel (vegetable oil and diesel blends) sprays in conditions close to diesel combustion engine. The dependency of the probability density distribution of droplet diameters on Weber number was analysed at momentum and energy losses $\bar{G}_{e}=0$ and $\bar{G}_{m v}=-0.017$ correspondently. The distribution of droplet diameters in biofuel was calculated at $\bar{G}_{e}=\bar{G}_{m v}$. The theoretical distributions show that, as a rule, the biodiesel produces a higher number of larger droplets than diesel fuel. In general the model is matching experimental data with reasonable accuracy.

\section{Acknowledgements}

The authors gratefully acknowledge the contribution of Ministry of Education of Ukraine to this research project by providing financial support. Also we would like to thank Prof. Christophe Dumouchel for his support in providing information about maximum entropy method.

\section{Nomenclature}

$D \quad$ droplet diameter $[\mu \mathrm{m}]$

$f \quad$ probability density function $\left[\mu \mathrm{m}^{-1}\right]$

We Weber number [-]

$\dot{n} \quad$ droplet generation rate $\left[\mathrm{s}^{-1}\right]$

$\dot{m}_{O} \quad$ mass flow rate $\left[\mathrm{kg} \mathrm{s}^{-1}\right]$

$j_{O}$ momentum $\left[\mathrm{kg} \mathrm{m} \mathrm{s}^{-2}\right]$

$\dot{E}_{O} \quad$ energy source introducing into a system $\left[\mathrm{J} \mathrm{s}^{-1}\right.$ ]

$\bar{G}_{m}, \bar{G}_{m N}, \bar{G}_{e}$ dimensionless sources for mass, momentum and energy [-]

$\sigma \quad$ surface tension $\left[\mathrm{N} \mathrm{m}^{-1}\right]$

$\rho \quad$ density of liquid phase $\left[\mathrm{kg} \mathrm{m}^{-3}\right]$

$\bar{V} \quad$ dimensionless droplet volume

$\bar{u} \quad$ dimensionless droplet velocity

$U_{0} \quad$ mean nozzle exit velocity $\left[\mathrm{m} \mathrm{s}^{-1}\right]$

$V_{m} \quad$ mean volume of droplets $\left[\mathrm{m}^{3}\right]$

$k_{B} \quad$ Boltzmann constant

$\alpha_{1,2,3}$ Lagrange multipliers

\section{References}

[1] Dumouhel, C. 2009, Entropy, 11, pp.713-747.

[2] "Handbook of Atomization and Sprays. Theory and Applications", 2011, edited by N. Ashgriz, Springer.

[3] Sovani, SD., Sojka, PE., Sivathanu, YR. 1999, Atomization Sprays, 9, pp. 113-152.

[4] Movahednejad, E., Ommi, F., Hosseinalipour, SM., 2010, Entropy, 12, pp.1484-1498.

[5] Sazhin, S., 2014, "Droplets and Spray". Springer.

[6] Yan, K., Ning, Z., Lü, M., Sun, C. 2015, Entropy, 17, pp. 580-593.

[7] Crua, C, Heikal, MR., Gold, MR., 2015, Fuel, 157, pp.140-150. 
[8] Crua, C., de Sercey, G, Heikal M.R., 2012, Sep. 2.-6., ICLASS, 12th Triennial International conference on Liquid Atomization and Spray System.

[9] Knothe, G., 2010, Progress in Energy and Combustion Science, 36, pp. 364-373.

[10] Yongyingsakthavorn, P., Vallikul, P., Fungtammasan, B., Dumouchel, C., Joulain, P., Dec. 1.-3., 2004, The Joint International Conference on "Sustainable Energy and Environment (SEE)".

[11] Kolodnytska, R., Al Qubeissi, M., Sazhin, S.S., 2013, ILASS, 25th European Conference on Liquid Atomization and Spray Systems.

[12] Kolodnytska, R.V. 2010, Visnik SNU, 6 (148), pp. 41-46.

[13] Ivashchenko A.V., Goryachkin V.N., 2011, Internal combustion engines, 2(41), pp. 41-46.

[14] Kolodnytska, R.V., Karimi, K., Crua, C., Heikal M.R., Sazhina, E.M. ,2008, Internal combustion engines, 1. pp. 42-46.

[15] Desantes, J.M., Payri, R., Garcıa, A., Manin, J., 2009, Energy \& Fuels, 23, pp. 3227-3235.

[16] Kamrak, J., Lorturn, P., Grehan, G., Saengkaew, S., Charinpanitkul, T., 2007, Aug 26.-29. The 5th Asian Aerosol Conference.

[17] Park, S.H., Kim H.J., Suh H. K., Lee C. S., 2009, International Journal of Heat and Fluid Flow, 30, pp. 960-970. 